\title{
Detection performance in pop-out tasks: Nonmonotonic changes with display size and eccentricity
}

\author{
Cristina Meinecke \\ Institut für Psychologie, Ludwig-Maximilians-Universität München, Leopoldstrasse 13, D 80802 München, \\ Germany

\section{Mieke Donk} \\ Vrije Universiteit Amsterdam, van der Boechorststraat 1, NL 1081 BT Amsterdam, The Netherlands; \\ and Max-Planck Institute for Psychological Research, Amalienstrasse 33, D 80799 München, Ger- \\ many \\ Received 22 February 2001, in revised form 10 December 2001
}

\begin{abstract}
We carried out three experiments to investigate detection performance in pop-out tasks and analysed how performance varied as a function of display size (number of elements) and retinal eccentricity of the target. Results showed that when display size was increased from 2 to 81 elements performance first decreased and then increased (replicating Sagi and Julesz, 1987 Spatial Vision 2 39-49). Performance variations differed as a function of eccentricity and often were more pronounced in the periphery than in the foveal area. This retinal-eccentricity influence suggests that processes underlying detection performance in small display sizes are different from those in large display sizes. One should be careful when using the variation of display size as an instrument to analyse visual-search processes because this analysis could be based on a comparison between non-equivalent conditions.
\end{abstract}

\section{Introduction}

When studying the functions of the visual system in the context of visual-search tasks, one widely applied manipulation is to vary the number of elements in a display as a diagnostic tool for differentiating between serial and parallel processing. Typically, when detection performance is independent of the number of elements in a display, performance is assumed to be based on parallel processing, whereas a (marked) decrease in performance with an increasing number of elements in a display is taken as an indicator for serial processing (eg Treisman and Gelade 1980; Treisman and Sato 1990; Wolfe et al 1989; but see Townsend 1974, 1990).

The present study focuses on the following task: a single line with a specific orientation is embedded within an array of lines taking the orthogonal orientation. This is a so-called 'pop-out' stimulus that, according to Wolfe (1992), generates parallel visual search. In the array of an (imaginary) $9 \times 9$ cell matrix, we varied the number of elements, starting with 2 and progressing until we reached a completely filled matrix with $9 \times 9=81$ elements. Participants had to report the presence or absence of a targeta line with an orientation orthogonal to the background lines. Detection performance was measured as a function of display size, as usual in visual-search tasks.

A basic assumption is that varying the number of elements in a display does not, per se, have any influence on the specific type of process that is active during visual search. Alternatively, the type of processing may change as a function of display size. This may even be very likely, when it is considered that increases in display size typically covary with increases in spatial density and/or increases in the size of the stimulus array. And these factors can exert an influence on detection performance (cf, eg, Carrasco et al 1995; for the influence on localisation performance cf, eg, Carrasco and Chang 1995). 
The literature provides evidence that detection performance even in a pop-out task actually does change as a function of display size. The first evidence comes from studies on visual search demonstrating a nonmonotonic relationship between display size and visual-search performance. For example, Carrasco and Frieder (1997) showed that when observers searched for a line among lines of a different orientation, 'convex' and 'concave' patterns of search functions were found (see, for similar findings, Carrasco et al 1998). Likewise, Sagi and Julesz (1987; see also eg Bacon and Egeth 1991; Sagi 1990) showed that increasing the display size from 2 to 81 elements can lead to an initial decrease in performance, but that this decrease can invert into an increase when the display size is further increased. How can these patterns of results be explained?

A performance decrease (with increasing display size) in pop-out tasks can be explained by invoking the mechanism of lateral inhibition or lateral masking (Bouma 1970; Huckauf et al 1999; see also the 'crowding effect', eg Strasburger et al 1991). Search is assumed to be parallel, but an increase in display size will reduce the distance between the single elements. Reducing the distance will increase the lateralmasking effect, thus leading to a decrease in the perception of the single elements. Sagi and Julesz (1987), however, in accordance with, eg, Wolfe et al (1989), assumed that a performance decrement, even with so-called pop-out stimuli, is an indicator of a serial-search process where an attentional mechanism is deployed serially.

An increase in performance is attributed usually to the associated increase in element density. Sagi and Julesz (1987) assumed that the visual system detects texture gradients between the target and the background elements (see also Nothdurft 1985, 1990; Nothdurft et al 1999), and that this detection of a gradient is easier when the distance between target and background elements is small. Bacon and Egeth (1991), on the other hand, assumed that the density of the background elements alone is the critical variable for a performance increase, and they postulated a mechanism of grouping by proximity.

In cases in which a mixture of these two patterns of results is found (decrease and increase), one could assume - in agreement with Carrasco and Frieder (1997) - that performance is a "function of particular ranges of set sizes" (page 69). In displays with a small number of elements, mechanisms such as lateral masking may be responsible for performance decrement; in displays with a large number of elements, grouping processes may be active and improve detection performance.

A second source of evidence that detection performance may change as a function of display size stems from comparisons of results in the context of visual-search tasks and texture-segmentation tasks. One of the typical differences is for display size to be relatively small in visual-search arrays, but relatively large in texture-segmentation arrays. Although some studies have suggested that the processes of parallel searching and effortless segmenting are the same, or at least behave just as if they were the same, Wolfe (1992) presented first evidence that parallel searching and effortless segmenting may be based on different processes. He demonstrated that parallel search can occur with stimuli that do not support effortless texture segmentation and vice versa. He concluded that texture segmentation and parallel visual search are not two measures of a single underlying mechanism.

A third source of evidence that a manipulation of the display size may influence detection performance comes, once again, from the comparison between studies on visual search and texture segmentation, but, this time, with respect to target eccentricity. In visual search, performance is usually best in foveal vision while declining with increasing retinal eccentricity of the target (eg Carrasco and Frieder 1997; Carrasco et al 1998; Donk 1995; Geisler and Chou 1995; Wolfe et al 1998). This decrease is explained through acuity limitations in the periphery (compared with foveal regions) and increasing lateral inhibition/masking with increasing eccentricity (Bouma 1970; 
Huckauf et al 1999). In texture segmentation, on the other hand, performance can improve with increasing eccentricity. This improvement is observed, for example, with stimuli in which orientation difference is the critical feature for discriminating between target and background elements, and it is observed at eccentricities between 3 and 6 deg [central performance drop (CPD): Gurnsey et al 1996; Joffe and Scialfa 1995; Kehrer 1987, 1989; Meinecke and Kehrer 1994; Yeshurun and Carrasco 1998]. This peripheral performance increase seems to be a consequence of the increasing size of receptive fields. Kehrer (1997) presented evidence for this explanation within the framework of a computational model.

These two opposite patterns of detection-performance outcomes as a function of retinal eccentricity in visual-search arrays and in texture arrays might be responsible for the nonmonotonic relation between increasing display size and detection performance found by, for example, Sagi and Julesz (1987): adding elements to an array initially impairs the detection of targets in peripheral areas because of the increasing effect of lateral masking; further addition of elements allows an improved detection of targets in peripheral areas because the arrays become more and more like texture arrays.

In sum, there is some evidence that pop-out stimuli are processed in a different way depending on the display size. This issue deserves attention, because display size variations are used as a diagnostic tool in studying the functions of the visual system. When detection performance is (nearly) independent of the number of elements in the display, performance is assumed to be based on parallel processing, an indicator for pop-out stimuli and feature search; when detection performance decreases with increasing number of elements in the display, performance is assumed to be based on serial processing; this serial processing is taken as an indicator for the involvement of an attentional mechanism which is necessary to detect targets that do not differ in a single simple feature from the background elements.

The aim of the present study was to analyse performance variations when detecting a feature target embedded in a varying number of background elements. We assumed that although all stimuli are pop-out stimuli, they are processed in a different way. To obtain more information on the underlying processes, detection performance was measured as a function of target eccentricity. As noted above, studies utilising relatively small display sizes - visual-search studies-demonstrate typically that performance decreases as a function of retinal eccentricity; whereas studies utilising large display sizes - texture-segmentation studies - show a substantial improvement of performance at eccentricities between 3 and 6 deg compared with the foveal and with more peripheral areas.

\section{Experiment 1}

In experiment 1 participants had to detect a target line of a specific orientation presented against a background of a variable number of lines with an orthogonal orientation. The target could appear at the centre of the display or at three different eccentricities. To ensure that performance was not at ceiling, we introduced a mask after the short presentation of the stimuli. Detection rates were used as major dependent variable. Additionally, search times were recorded to rule out a possible speed-accuracy trade-off.

Our research questions were: (a) Can we replicate the findings of Sagi and Julesz (1987) showing a nonmonotonic relation between increasing display size and detection performance? (b) When analysing performance as a function of the retinal eccentricity of the target, can we find a performance decrease (with increasing eccentricity) when display size is small, and a performance increase (again with increasing eccentricity) when display size is large? 


\subsection{Method}

2.1.1 Participants. Nine participants (eight female) were paid to participate in the experiment. They were aged 21-35 years (mean: 27 years). All participants (in all the experiments reported here) had normal or fully corrected visual acuity (this was tested with a Rodenstock R12 Vision Tester; Test stimuli No. 112).

2.1.2 Apparatus. The experiment was controlled by an HP Vectra QS/20 computer, and stimuli were presented on an NEC Multi Sync 4FG screen. Each participant sat at a table on which a head-and-chin rest was mounted. The display monitor was positioned to give an observation distance of $40 \mathrm{~cm}$ with the direction of gaze inclined slightly downward. Participants responded to the stimuli by pressing the left or right mouse key.

2.1.3 Stimuli. The background elements were diagonal lines inclined $45^{\circ}$ to the left; the target element was a diagonal line inclined $45^{\circ}$ to the right (orientation difference between target and background lines: $\left.90^{\circ}\right)$. The lines were $18 \mathrm{~mm}(2.58 \mathrm{deg})$ long and $3.3 \mathrm{~mm}(0.47 \mathrm{deg})$ broad and were arranged in a $9 \times 9$ element matrix $(166.5 \mathrm{~mm}$, $24 \mathrm{deg}$ ). The minimum distance between adjacent lines in a horizontal and vertical direction (measured from line centre to line centre) was $19.25 \mathrm{~mm}$ (2.75 deg). Elements were displayed in black against a screen background of white/gray $\left(10.0 \mathrm{~cd} \mathrm{~m}^{-2}\right)$. The position of the imaginary matrix was centred on the screen.

On one half of the trials, the display contained a target. This could appear in all cells lying on the two diagonals of the matrix except for the outer border cells. This resulted in 13 different target positions: one position in the centre of the matrix ( 0 deg eccentricity), and three positions on each of the four diagonal branches $(4,8$, $12 \mathrm{deg}$ eccentricity). The position of the target was restricted to these cells to ensure enough data for each eccentricity.

The mask consisted of superimposed target and background lines on each cell of the $9 \times 9$ matrix. There were five display-size conditions (2, 7, 21, 49, and 81 elements). Elements were placed in the matrix randomly. An example of a stimulus with 49 elements is given in figure 1.

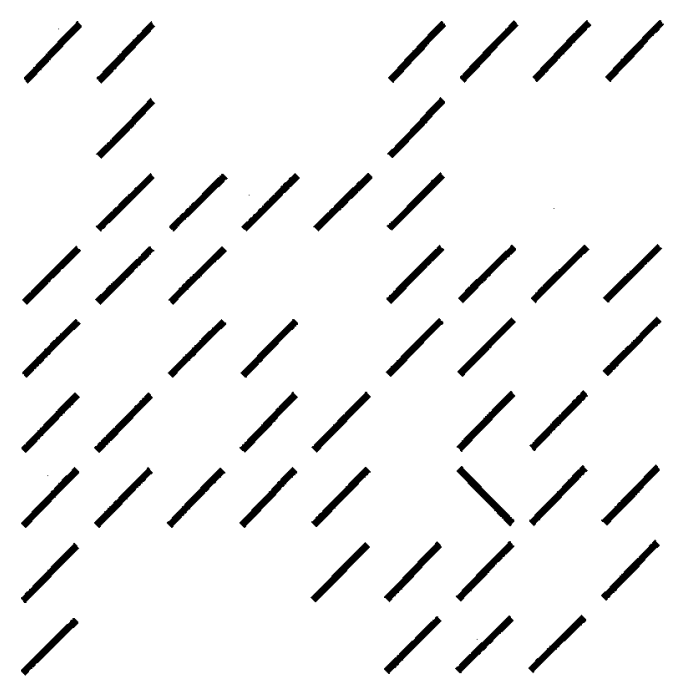

Figure 1. Experiment 1. Example of a stimulus (with target) of display size ' 49 '.

2.1.4 Procedure. A within-subject design was used. Target eccentricity (0, 4, 8, 12 deg) and target presence (present versus absent) were varied randomly within blocks.

To allow participants to adjust themselves to each display-size condition $(2,7,21$, 49, 81 elements), these were blocked, and the sequence of presentation was determined according to a Latin square. 
A block started with three randomly chosen trials that were not included in the data analysis. Afterwards, 32 negative trials, in which the stimulus matrix contained no target, and 32 positive trials, in which a target was presented twice at each possible position $(0,4,8,12 \mathrm{deg})$ of the four diagonal branches, were presented at random. Four blocks were presented in each display-size condition.

Each trial started with the presentation of a tone $(5 \mathrm{kHz}, 200 \mathrm{~ms})$ followed immediately by the presentation of a central fixation point. After $511 \mathrm{~ms}$, the stimulus was presented for $83.5 \mathrm{~ms}$ and followed immediately by the mask. The mask remained on the screen until the participant responded. If the response was correct, the next trial started. If the response was wrong, the word "falsch" (wrong) appeared on the screen for $511 \mathrm{~ms}$, followed by the next trial.

Participants were instructed to fixate as closely as possible on the fixation point and to respond as quickly as they could while making as few false alarms as possible. The last point was introduced to keep false alarms to a minimum, so that a participant would give a "yes" response only if he or she was certain that the stimulus contained a target. The purpose of this instruction was to keep interindividual criterion differences as low as possible.

Each participant took part in one session lasting about $1 \mathrm{~h}$. The session started with a practice block of 320 trials that were not included in the data analysis. Participants were free to take a break between blocks.

\subsection{Results}

Figure 2 presents detection performance as a function of display size [(a) $d^{\prime}$ and (b) reaction time of hits], and the percentage of hits as a function of eccentricity and display size (c). An analysis of variance for repeated measures was calculated separately for the different data types.

$d^{\prime}$ data: Performance varies significantly with display size $(2,7,21,49,81$ elements) $\left(F_{4,32}=12.54, p<0.001\right)$.

Reaction time of hits: Reaction time increases with increasing display size. This variation, however, is not significant $(F<1)$.

Eccentricity data: Performance varies significantly with display size (2, 7, 21, 49, 81 elements) $\left(F_{4,32}=16.63, p<0.001\right)$, and with retinal eccentricity of the target $(0,4,8,12 \mathrm{deg})$ $\left(F_{3,24}=7.78, p<0.001\right)$.

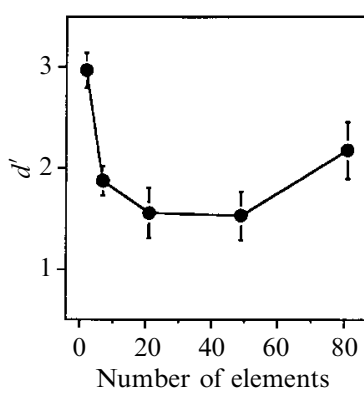

(a)

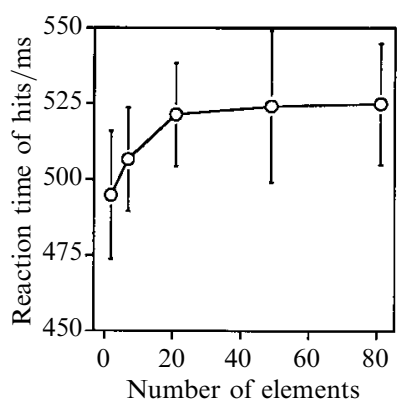

(b)

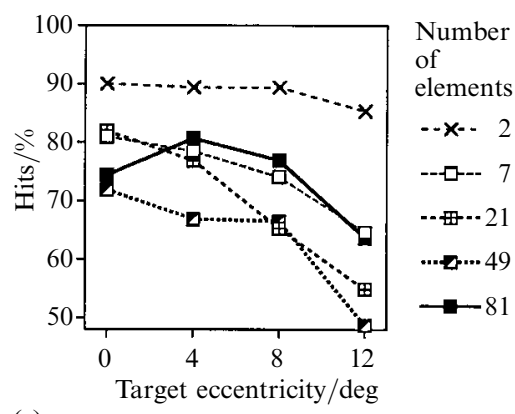

(c)

Figure 2. Experiment 1. Performance as a function of the number of elements on the display: (a) $d^{\prime}$, (b) reaction time of hits, (c) percentage of hits as a function of the retinal eccentricity of the target for each display-size condition.

The interaction between display size and eccentricity is significant $\left(F_{12,96}=2.27\right.$, $p<0.05)$. For each display-size condition, a separate regression analysis was calculated for the data as a function of eccentricity. For display-size conditions of 7 and 21 elements, linear regressions provide the best explanation of the data $(F=22.94$, $p<0.05 ; F=84.08, p<0.05$, respectively). 


\subsection{Discussion}

2.3.1 Display size. Results show that detection performance starts by decreasing with increasing display size and then increases between display-size conditions of 49 and 81 elements. The reaction-time data show that there is no speed-accuracy trade-off. Thus, the nonmonotonic performance function reported by Sagi and Julesz (1987) can be replicated. Unlike Sagi and Julesz, who observed an increase in performance over a wider range of display sizes, we found a clear increase only in the condition in which all the cells of the stimulus matrix were filled with elements. Experiment 2 analyses detection performance in conditions between display-size conditions of 49 and 81 elements in more detail.

It is interesting to note that even in conditions in which the display sizes are in a range typically used in visual-search experiments $(2,7,21$ elements), performance by no means remains unaffected by the manipulation of the display size. Unlike, for example, the data presented by Wolfe (1992), in which performance was independent of display size, our data show a performance decrease with increasing display size. We presume that with unlimited presentation time, as in many visual-search studies, performance could be at ceiling. If ceiling effects are avoided, one can observe the predicted influence of increased density of elements on detection performance.

2.3.2 Retinal eccentricity. As figure 2c shows, performance decreases with increasing eccentricity in almost all display-size conditions: with 2 elements on the display, the impairment is visible only slightly at the most eccentric position (12 deg), suggesting that performance was near ceiling in this condition. In display-size conditions of 7, 21, and 49 elements, however, there is a clear performance impairment with increased eccentricity. In display-size conditions of 7 and 21 elements, this impairment follows a linear function, as predicted by the lateral-masking effect; in display-size condition of 49 elements, the linearity is not 'perfect,' suggesting that a change in the processing mode is initiated here.

The data show further that the more elements on the display, and thus the more dense the arrangement, the stronger the influence of eccentricity on detection performance. This pattern of results is in accordance with visual-search findings (cf Carrasco and Frieder 1997; Carrasco et al 1995) and can be explained by the lateral-masking effect.

In display-size condition of 81 elements, in which all cells of the matrix are filled with elements, detection performance as a function of eccentricity takes a completely different course. Here, we find the typical CPD curve (see Kehrer 1987) reported in texture-segmentation tasks: starting from the foveal region, performance increases with increasing eccentricity. It is only at further eccentricities that performance declines. ${ }^{(1)}$ At position $0 \mathrm{deg}$ that is when the target is presented at the centre of the display, performance is nearly identical in display-size conditions of 49 and 81 elements. Thus, the performance increase between display-size conditions of 49 and 81 elements is apparently due completely to the better ability of the observers to detect an eccentric target when the matrix is completely filled with elements (ie with 81 elements). ${ }^{(2)}$

\section{Experiment 2}

In experiment 2, we wanted to fill the gap between display-size conditions of 49 and 81 elements. On the basis of the results in experiment 1, it could not be determined whether the peripheral benefit of 81 elements relied on the special case of the completely

(1) Note that a CPD curve is not a result of criterion shifts, as shown by Kehrer (1989).

(2) A comparison of display-size condition 49 with display-size condition 81 casts light on the nature of the 'central performance drop': it seems appropriate to rename this effect a peripheral performance increase (performance increases up to 3 to $6 \mathrm{deg}$ of retinal eccentricity, before it decreases again), because this reflects the true nature of the change in performance. 
filled matrix or whether this change would be observed gradually when the display size is increased stepwise from 49 to 81 elements. Therefore, in experiment 2 we used five display-size conditions: $49,57,65,73$, and 81 elements.

We presume that the effect is not a specificity of display-size condition of 81 elements. Sagi and Julesz (1987) found an increase in detection performance not only at the largest display size, but already at smaller display-size conditions. If we could replicate this finding, the interesting question would be how detection performance relates to retinal eccentricity.

\subsection{Method}

3.1.1 Participants. Eight participants (five female) were paid to participate in the experiment. They were aged $22-37$ years (mean: 28.3 years).

\subsubsection{Apparatus. This was identical to that in experiment 1.}

3.1.3 Stimuli. These were identical to the stimuli in experiment 1 except for the use of the following display sizes: $49,57,65,73$, and 81 elements.

3.1.4 Procedure. Two sessions were administered. In the first session, five blocks per display size were presented, each block consisting of $2 \times 32$ positive trials and $2 \times 32$ negative trials. In the second session, a further five blocks were presented, but these blocks consisted of $3 \times 32$ positive trials and $3 \times 32$ negative trials. All other details were identical to experiment 1 .

\subsection{Results}

Figure 3 presents detection performance as a function of display size [(a) $d^{\prime}$ and (b) reaction time of hits], and the percentage of hits as a function of eccentricity and display size (c). An analysis of variance for repeated measures was calculated separately for the different data types.

$d^{\prime}$ data: Performance varies significantly with display size (49, 57, 65, 73, 81 elements) $\left(F_{4,28}=6.94, p<0.005\right)$.

Reaction time of hits: Reaction time decreases with increasing display size $\left(F_{4,28}=3.18\right.$, $p<0.05)$.

Eccentricity data: Performance varies significantly with display size (49, 57, 65, 73, 81 elements $)\left(F_{4,28}=4.92, p<0.005\right)$, and eccentricity of the target $(0,4,8,12 \mathrm{deg})$ $\left(F_{3,21}=3.43, p<0.05\right)$.

The interaction between display size and eccentricity was significant $\left(F_{12,84}=2.35\right.$, $p<0.05)$. Regression analysis for detection performance as a function of eccentricity for the different display-size conditions showed that the data could not be explained with linear regressions (all cases: quadratic or higher).

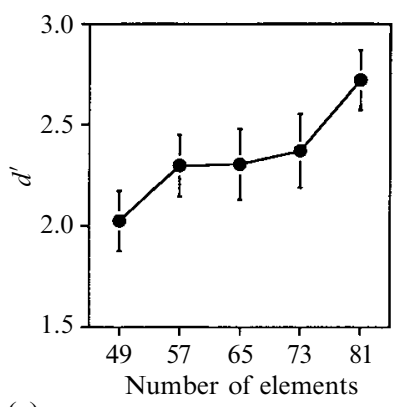

(a)

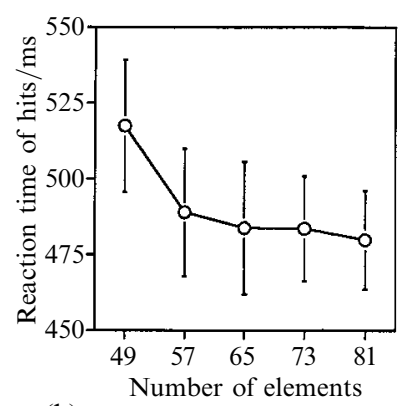

(b)

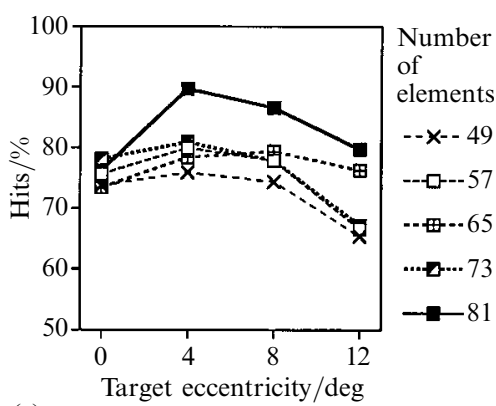

(c)

Figure 3. Experiment 2. Performance as a function of the number of elements on the display: (a) $d^{\prime}$, (b) reaction time of hits, (c) percentage of hits as a function of the retinal eccentricity of the target for each display-size condition. 


\subsection{Discussion}

The results of experiment 2 show an increase in performance as display size increases. This agrees well with the findings of Sagi and Julesz (1987) and Bacon and Egeth (1991); thus the increase in detection performance with 81 elements in experiment 1 is not special and unique. Furthermore, the regression analysis shows that detection performance is not linearly related to retinal eccentricity. In other words, in none of the realised display-size conditions performance decreases steadily as a function of eccentricity. Rather, performance first improves (more or less, depending on the display-size condition) and only with further eccentricity deteriorates. Performance increase is particularly pronounced with 81 elements. As stated before, display-size condition of 81 elements has a property that is unique to this condition: It is the only condition in which all cells of the matrix are filled with elements. This might be a special case of a stimulus, as Carrasco et al (1998) have already pointed out: the high regularity of the stimulus might facilitate the detection of the target - the only discontinuity. Experiment 3 will analyse this in more detail.

\section{Experiment 3}

In experiment 3, we compared detection performance in display-size condition of 81 elements with performance in a condition in which one matrix cell was kept empty (display-size condition of 80 elements). If the fact that all the cells of the matrix are filled with elements is responsible for the good performance with 81 elements, then one empty cell should have a visible effect on performance.

\subsection{Method}

4.1.1 Participants. Nine participants (eight female) were paid to participate in the experiment. They were aged $20-32$ years (mean: 24.3 years).

\subsubsection{Apparatus. This was identical to that in experiment 1.}

4.1.3 Stimuli. These were identical to the stimuli in experiment 1 except that only two display sizes were realised: 80 and 81 elements.

4.1.4 Procedure. One session was administered. Six blocks per display-size condition were presented, each block consisting of 32 positive and 32 negative trials. Stimuli were presented for $66 \mathrm{~ms}$; this reduction in presentation time was introduced to ensure that no ceiling effect could occur. All other details were identical to experiment 1.

\subsection{Results}

Figure 4 presents detection performance as a function of display size [(a) $d^{\prime}$ and (b) reaction time of hits], and the percentage of hits as a function of eccentricity and display size (c). An analysis of variance for repeated measures was calculated separately for the different data types.

$d^{\prime}$ data: Performance varies significantly with display size (80 versus 81 elements) $\left(F_{1,8}=7.11, p<0.05\right)$.

Reaction time of hits: Reaction time decreases with increasing display size; however, this decrease is not significant $\left(F_{1,8}=1.06, \mathrm{~ns}\right)$.

Eccentricity data: Performance varied significantly with display size ( 80 versus 81 elements) $\left(F_{1,8}=8.01, p<0.05\right)$. Performance varied with retinal eccentricity of the target $(0,4$, $8,12 \mathrm{deg})$, but just failed to reach significance $\left(F_{3,24}=2.99, p=0.051\right)$.

There was no significant interaction between display size and eccentricity $\left(F_{3,24}=0.06, \mathrm{~ns}\right)$. 


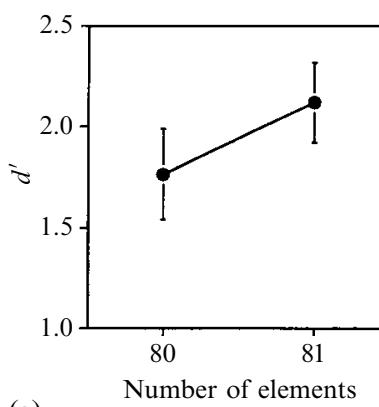

(a)

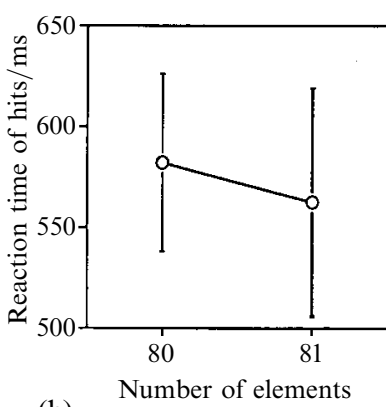

(b)

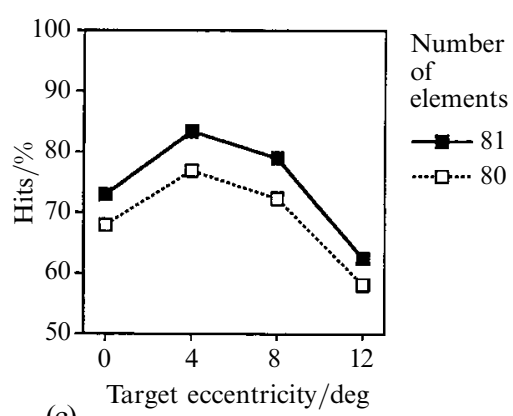

(c)

Figure 4. Experiment 3. Performance as a function of the number of elements on the display: (a) $d^{\prime}$, (b) reaction time of hits, (c) percentage of hits as a function of the retinal eccentricity of the target for each display-size condition.

\subsection{Discussion}

There is a clear difference between display-size conditions of 80 and 81-elements: performance declines when one single cell of the $9 \times 9$ matrix is left empty. In both conditions, a typical CPD curve can be observed. But, unlike experiments 1 and 2, in which a significant interaction between display size and eccentricity was found, experiment 3 reveals no interaction (figure $4 \mathrm{c}$ ): the difference between both conditions is merely a difference in the level of performance. We shall consider this result in more detail in the next session.

\section{General discussion}

The aim of the present study has been to analyse detection performance in pop-out stimuli, specifically to look at performance variations as a function of display size. An implicit assumption in visual-search experiments is that varying the number of elements in a display does not, per se, have any influence on the type of process responsible for detection performance. The pattern of results in our experiments suggests that the processes underlying detection performance in small display sizes differ from those processes underlying detection performance in large display sizes. ${ }^{(3)}$ Although all stimuli may be classified as typical pop-out stimuli, we can observe rather strong variations in performance. In agreement with Sagi and Julesz (1987), performance shows a nonmonotonic relation with increasing display size. Increasing the number of background elements leads to an initial decrease in performance. However, when all the cells of the stimulus matrix are filled with elements (display-size condition of 81 elements), performance increases markedly (experiment 1). This increase in performance can be observed not only in the condition with a completely filled matrix but also with display sizes between 49 and 81 elements (experiments 2 and 3 ).

These performance variations differ as a function of eccentricity and are-in some cases-more pronounced in the periphery than in the fovea. Performance decreases, eg from display-size condition of 2 to 49 elements, more on peripheral positions than in the foveal position; and the observed performance increase between condition of 49 and 81 elements is nearly solely due to an increase on peripheral positions (cf figure 2).

When display size is relatively small, a performance decrease is found when display size is increased and when the retinal eccentricity of the target is increased. This pattern of results has to be expected when the single elements form the basis for perception, because of acuity limitations and because of the increased lateral-masking effect with increasing density and with increasing eccentricity (eg Bouma 1970). One might call this the single-element hypothesis.

(3) Although we do not claim that display size is the critical variable, as we will show we assume that other stimulus properties which are a consequence of varying display size may be responsible for the observed performance variations. 
When display size is relatively large, then a performance increase is found when density is increased and when retinal eccentricity is increased (from the fovea up to about 4 deg eccentricity, decreasing again on more eccentric positions). Such a peripheral advantage has to be expected when some form of spatial integration forms the basis for perception, because a grouping of background elements (cf Bacon and Egeth 1991) and/or the detection of a texture gradient (cf Sagi and Julesz 1987) should profit from smaller inter-element distances and from larger receptive fields outside the fovea. Because receptive fields are larger in peripheral regions than in the foveal region, such an integration over space might be an (automatic) byproduct when the stimulus is projected onto peripheral regions. One might call this the spatial-integration hypothesis.

When all cells of the matrix are filled with elements, performance is particularly good. In combination with the results from a condition in which only one cell is left empty (experiment 3), one can infer a third process responsible for detection performance that relies on the target being an irregularity in an otherwise regular background. Display-size condition of 81 elements has a feature that is unique to this condition: all cells of the matrix are filled with identical elements. In trials with a target, this target deviates from an otherwise regular background. Thus, the target might be detected by a mechanism that is sensitive to irregularities. Evidence for this assumption comes from a study measuring event-related potentials (ERPs) in a texturesegmentation task (Schlaghecken et al 2001). This revealed that inhomogeneous textures (textures with target) were processed differentially from homogeneous textures (textures without target). Every time an inhomogeneous texture was presented, the P3 component in the ERP was enlarged. This P3 enlargement is usually found in the context of the oddball paradigm indicating the processing of the infrequent, deviant stimulus (cf eg Näätänen 1992; Schröger 1997). Schlaghecken et al (2001) suggested that the detection of a target in an otherwise homogeneous background may be described as the detection of a spatial oddball or irregularity.

If one assumes that the target in display-size condition of 81 elements is detected by an irregularity-detection mechanism, then the pattern of results in experiment 3 can be explained as follows: in display-size condition of 81 elements, if an irregularity is detected, this is always a valid indicator for the presence of a target. However, in display-size condition of 80 elements, if an irregularity is detected, this could be a target or an empty cell in the matrix. In this condition, participants have to decide whether the irregularity in the actual stimulus is the task-relevant 'correct' irregularity (line of a different orientation) or a task-irrelevant irregularity (the empty cell). Sometimes, they make the wrong decision, producing more misses and more false alarms.

It is interesting that performance as a function of eccentricity takes the same course in both conditions. The presence of an empty cell exerts no differential influence on detection performance. This supports the view that the empty cell influences performance in a more general manner (as described above), and that it is not the perception of the target per se that is impaired.

Thus, one may conclude from the results in experiment 3 that irregularities in the stimulus array may influence detection performance in this sort of task. One might call this the irregularity-detection hypothesis.

The results further suggest that stimulus properties interact with properties of the visual system. The fovea is best at analysing detailed information. With stimuli that allow an effortless analysis of their single elements (displays with low density), performance is best in the foveal region and declines with eccentricity. The periphery is best at analysing global properties of the stimuli, properties that need spatial integration. With stimuli that allow effortless integration of information over space (displays with high density), performance is best not in the fovea, but in peripheral regions 
(about 3-6 deg eccentricity). The results agree with a model presented by Meinecke (1989; cf also Meinecke et al 2002) that assumes different 'grains' of analysis at different retinal eccentricities.

In sum, our results support the view that, even in a pop-out task, processes underlying detection performance in small display sizes differ from those underlying detection performance in large display sizes. As a consequence, in accordance with Carrasco et al (1995) and Carrasco and Frieder (1997), it is doubtful whether variation of display size can be used as a major diagnostic tool for determining whether parallel or serial processes are active. Although we have found variations in performance as a function of display size, we would not conclude from our results that these variations are indicators for some form of serial processing. We claim that all our stimuli are processed in parallel, but that, as a function of display size, different processes are responsible for detection performance. These different processes originate in constraints of the visual system (eg receptive field sizes) in interaction with stimulus properties (eg increased density). We propose three types of process that still require closer examination and verification.

One might argue that, in the context of visual-search experiments, the distinction between parallel and serial processing has lost its validity and that it makes more sense to speak, eg, of different degrees of efficiency (Wolfe 1998). The data of visual-search experiments in the last decades support this change in terminology when describing search performance. But even in the 'efficiency' context, the search analysed in our study usually is categorised as 'very efficient', as we used one of the classical pop-out tasks, with orientation difference as the critical feature between target and context elements. Nevertheless, we could observe rather pronounced performance variations with variation of the display size. Should we classify our observed search performance (at least when looking at the performance decrease) as 'rather inefficient'? Our claim is a different one: performance must change when the number of elements in a display is varied, because several other factors covary with this manipulation (eg density and/or eccentricity, regularity), and because we know that these factors exert an influence on detection performance. Thus, comparing search performance in displays with few elements with performance in displays with many elements may be a comparison between non-equivalent conditions, a comparison between conditions where several factors vary simultaneously. One should be careful to use the variation of display size (number of elements) as an instrument to analyse visual-search processes. Our data suggest that, indeed, the visual system treats the different display conditions differently, applying different processing modes when solving the task to detect the target.

Acknowledgments. We are grateful to Christina Grandegger and Silvia Bauer for conducting the experiments and Kai Engbert for his assistance in analysing the data, and we wish to thank Jonathan Harrow for correcting our English style. We would like to thank Marisa Carrasco for her helpful comments on an earlier version of the manuscript.

\section{References}

Bacon W F, Egeth H E, 1991 "Local processes in preattentive feature detection" Journal of Experimental Psychology: Human Perception and Performance 17 77-90

Bouma H, 1970 "Interaction effects in parafoveal letter recognition" Nature 226 177-178

Carrasco M, Chang I, 1995 "The interaction of objective and subjective organizations in a localization search task" Perception \& Psychophysics $571134-1150$

Carrasco M, Evert D L, Chang I, Katz S M, 1995 "The eccentricity effect: Target eccentricity affects performance on conjunction searches" Perception \& Psychophysics $571241-1261$

Carrasco M, Frieder K S, 1997 "Cortical magnification neutralizes the eccentricity effect in visual search" Vision Research $3763-82$

Carrasco M, McLean T L, Katz S M, Frieder K S, 1998 "Feature asymmetries in visual search: Effects of display duration, target eccentricity, orientation and spatial frequency" Vision Research $38347-374$ 
Donk M, 1995 "Some evidence for unequal loss of location and feature information as a function of retinal eccentricity in visual search" Visual Cognition $2201-220$

Geisler W S, Chou K-L, 1995 "Separation of low-level and high-level factors in complex tasks: Visual search" Psychological Review 102 356-378

Gurnsey R, Pearson P, Day D, 1996 "Texture segmentation along the horizontal meridian: Nonmonotonic changes in performance with eccentricity" Journal of Experimental Psychology: Human Perception and Performance $22738-757$

Huckauf A, Heller D, Nazir T, 1999 "Lateral masking: Limitations of the feature interaction account" Perception \& Psychophysics $61177-189$

Joffe K M, Scialfa C T, 1995 "Texture segmentation as a function of eccentricity, spatial frequency and target size" Spatial Vision $9325-342$

Kehrer L, 1987 "Perceptual segregation and retinal position" Spatial Vision 2 247-261

Kehrer L, 1989 "Central performance drop on perceptual segregation tasks" Spatial Vision 4 45-62

Kehrer L, 1997 "The central performance drop in texture segmentation: A simulation based on a spatial filter model" Biological Cybernetics 77 297-305

Meinecke C, 1989 "Retinal eccentricity and the detection of targets" Psychological Research 51 107-116

Meinecke C, Kehrer L, 1994 "Peripheral and foveal segmentation of angle textures" Perception \& Psychophysics 56 326-334

Meinecke C, Kimchi R, Grandegger C, 2002 "A reversal in the direction of detection asymmetry: Effects of spatial density, spatial regularity, and retinal eccentricity" Perception \& Psychophysics (in press)

Näätänen R, 1992 Attention and Brain Function (Hillsdale, NJ: Lawrence Erlbaum Associates)

Nothdurft H C, 1985 "Sensitivity for structure gradient in texture discrimination tasks" Vision Research $251957-1968$

Nothdurft H C, 1990 "Texton segregation by associated differences in global and local luminance distribution" Proceedings of the Royal Society of London, Series B 239295 -320

Nothdurft H C, Gallant J L, Van Essen D C, 1999 "Response modulation by texture surround in primate area V1: Correlates of 'popout' under anesthesia" Visual Neuroscience 1615 - 34

Sagi D, 1990 "Detection of an orientation singularity in Gabor textures: Effect of signal density and spatial-frequency" Vision Research 301377 - 1388

Sagi D, Julesz B, 1987 "Short-range limitation on detection of feature differences" Spatial Vision $239-49$

Schlaghecken F, Meinecke C, Schröger E, 2001 "Processing spatial and temporal discontinuities: Electrophysiological indicators" Journal of Psychophysiology $1580-94$

Schröger E, 1997 "On the detection of auditory deviants: A pre-attentive activation model" Psychophysiology $34245-257$

Strasburger H, Harvey L O J, Rentschler I, 1991 "Contrast thresholds for identification of numeric characters in direct and eccentric view" Perception \& Psychophysics 49 495-508

Townsend J T, 1974 "Issues and models concerning the processing of finite number of inputs", in Human Information Processing: Tutorials in Performance and Cognition Ed. B H Kantowitz (Hillsdale, NJ: Lawrence Erlbaum Associates) pp 133-168

Townsend J T, 1990 "Serial vs parallel processing: Sometimes they look like Tweedledum and Tweedledee, but they can (and should) be distinguished" Psychological Science 1 46-54

Treisman A M, Gelade G, 1980 "A feature-integration theory of attention" Cognitive Psychology $1297-136$

Treisman A M, Sato S, 1990 "Conjunction search revisited" Journal of Experimental Psychology: Human Perception and Performance $16459-478$

Wolfe J M, 1992 "'Effortless' texture segmentation and 'parallel' visual search are not the same thing" Vision Research $32757-763$

Wolfe J M, 1998 "Visual search", in Attention Ed. H Pashler (Hove, East Sussex: Psychology Press) pp $13-73$

Wolfe J M, Cave K R, Franzel S L, 1989 "Guided search: An alternative to the feature integration model for visual search" Journal of Experimental Psychology: Human Perception and Performance 15419 - 433

Wolfe J M, O'Neill P, Bennett S C, 1998 "Why are there eccentricity effects in visual search? Visual and attentional hypotheses" Perception \& Psychophysics $60140-156$

Yeshurun Y, Carrasco M, 1998 "Attention improves or impairs visual performance by enhancing spatial resolution" Nature $39672-75$ 